\title{
Quantitative Risk Assessment of LPG Tank Area
}

\author{
Hu Xinsheng ${ }^{1, \text { a }}$, Wu Zongzhi ${ }^{1,2, b}$, Xiao Lifang ${ }^{3, c}$ and Wang Rujun ${ }^{4,5, d}$ \\ ${ }^{1}$ Faculty of Resource and Safety Engineering, China University of Mining and Technology (Beijing), \\ Beijing 100083, China \\ ${ }^{2}$ The Occupational Safety and Health Administration, State Administration of Work Safety, Beijing \\ 100713, China \\ ${ }^{3}$ Daqing Oil Field Co., Petro China Co Ltd., Heilongjiang, 163000, China \\ ${ }^{4}$ China Academy of Safety Science and Technology, Beijing 100012, China \\ ${ }^{5}$ Key Laboratory of Major Hazard control and Accident Emergency Technology, State \\ Administration of Work Safety, Beijing 100012, China \\ a huxinsheng1989@126.com, buzongzhi@vip.sina.com, ${ }^{\text {c }}$ cassthu@163.com, ${ }^{d}$ \\ wangrj_oshi@163.com
}

Keywords: LPG, Accident scenarios, Consequence models, Individual risk

Abstract. In order to study the hazard of LPG storage, quantitative risk assessment is proposed to analyze the accident scenarios. The consequence models specific to the leak of LPG storage is put forward and the software Phast Risk is used to calculate the individual risk. The results of typical historical accidents show that BLEVE, vapor cloud explosion and flash fire are the major accident scenarios according to the different leakage apertures. Fireball model, TNT equivalent method, heavy gas dispersion model can be used to calculate the consequences of LPG leak. The case study shows that required minimum distances between hazards and vulnerable objects should be $600 \mathrm{~m}, 800 \mathrm{~m}, 900$ $\mathrm{m}$, corresponding to the low-density, high-density, and special high-density places, respectively. This study provides data support to determine the safety distance of the chemical plant and the surrounding environment from a risk management point of view.

\section{Introduction}

LPG is short for Liquefied Petroleum Gas that is stored in the pressured vessel. It is widely used in the industries because of less pollution. However, after mixed with air, the explosive mixture has the potential risk of fire and explosion. Therefore, the production and storage of LPG should be strict supervision, especially the LPG storage tank that would result in the accident if the leak occurs.

In order to prevent LPG accident, Quantitative risk assessment (QRA) can be used to calculate the potential risk of chemical engineering equipment and device [1]. The research object of this paper is the LPG storage tank. According to the procedure of QRA, the severest scenarios of LPG leak and the consequence models are analyzed, as well as the individual risk is calculated. Based on a case study of LPG spherical tank area, the software Phast Risk is used to complete the calculation and then the result is compared with the individual risk criteria. The research can provide references and suggestions for the risk conduct and the layout planning of the similar tank area.

\section{LPG Accident Scenarios}

Historical Accidents. The accident result from the LPG tank leak could lead to the heavy casualties and serious economic losses. The two historical accident below can explain the event sequence after the leak.

An LPG accident happened on November 16, 1984, in an LPG storage tank called PEMEX that located in Mexico City. The vapor dispersed to the slum that is $400 \mathrm{~m}$ far away from the factory and then was ignited. The instantaneous flash fire and the subsequent BLEVE (Boiling Liquid Expanding Vapor Explosion) cause huge fatalities. The congestion of the fire propagation path accelerated the fire 
propagation velocity, which resulted in a Vapor Cloud Explosion (VCE). This accident killed about 500 people and injured about 7000 people, besides the tank area was totally destroyed.

Another LPG accident happened in Shandong, China. July 16, 2015, an LPG spherical tank of a chemical engineering company was leaking. The vapor was ignited and the deflagration was happening. The spherical tank then occurred BLEVE and VCE. There was a huge mushroom cloud in the worksite. Buildings and walls collapsed in the explosion and the fire engulfed 9 spherical tanks. All the residents within the $5 \mathrm{~km}$ range were evacuated.

Event Sequences. The event sequence after LPG leak can be concluded according to the historical cases, shown in Fig. 1. After the LPG tank leak, the vapor cloud will encounter the ignition and the flash fire will first occur. Then the flash fire will travel back to the leak hole leads to a jet fire. The high temperature of the jet fire heats the pressured vessel and result in BLEVE. Besides, the VCE will happen if the fire propagation velocity increase because the late ignition of vapor cloud. The accident consequence is different according to the different ignition time and the leak hole. For the small leak, no matter the immediate or the late ignition, the consequence that could affect the farthest distance is BLEVE. For the large leak hole or the rupture, flash fire or the VCE result from late ignition could affect the farthest distance.

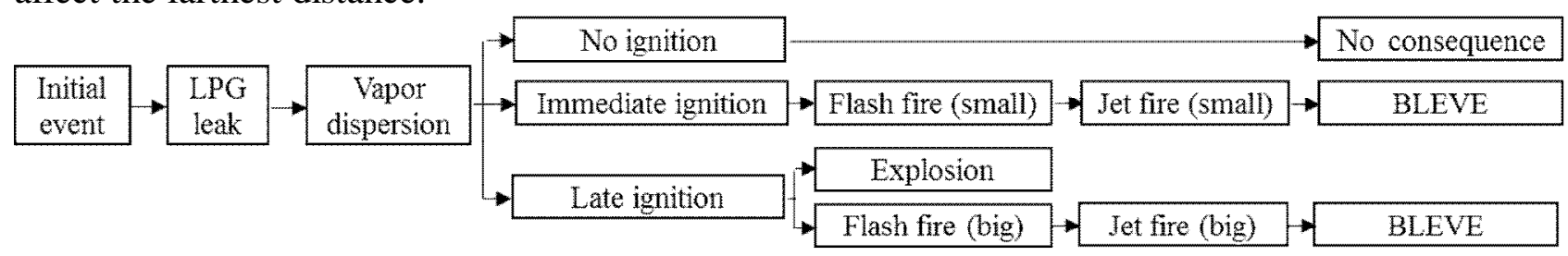

Fig. 1 Event sequences of LPG leak

Event tree analysis. LPG event tree was built according to the above historical cases and event sequences, shown in Fig. 2. The severest consequence of a small leak is BLEVE. For the large leak or the rupture, the severest consequence is flash fire or VCE.

\begin{tabular}{l|l|l|l|}
\hline (A) LPG & Ignition & BLEVE & Consequence \\
\multirow{5}{*}{ Small leak } & Yes & Yes & BLEVE \\
\cline { 2 - 4 } & No & No & No \\
& & & No
\end{tabular}

\begin{tabular}{l|l|l|l|}
\hline (B) LPG & Late ignition & Explosion & Consequence \\
& Yes & Yes & VCE \\
\cline { 3 - 4 } Large leak & \multicolumn{2}{|c}{ No } & Flash fre \\
\cline { 3 - 4 } or rupture & No & & No
\end{tabular}

Fig. 2 Event trees of LPG with small and medium leak (A), large leak or rupture (B)

\section{Consequence models}

BLEVE consequence model. The BLEVE explosion occurs in the LPG pressured storage tank. This is because the pressured vessel is cracked after being bake at a high temperature, at the same time the internal pressure medium is boiling that leading to volume expansion. As a result, the fireball explosion is occur. To calculate the consequence of BLEVE, the first step is to calculate fireball basic parameters including fireball diameter, burning time, lifting height, and then calculate the thermal radiation flux at the point of observation.

VCE consequence model. VCE will occur if LPG vapor cloud is ignited late and the fire propagation velocity is accelerated. TNT equivalent method is the classical model to simulate the consequence of VCE by transforming the destructive effect of the explosions into a damaging effect of the TNT explosion.

Flash fire consequence model. After LPG vapor cloud ignited, the flash fire will occur if the fire propagation velocity is not accelerated by the congestion on the propagation path. The duration of flash fire is relatively short and the shock wave is also small. Therefore, it will not result in the huge casualty for the people that outside the flame range. However, the people inside will die. The flame range related to the Low Flammability Limit (LFL). The consequence of flash fire is to calculate the vapor concentration at the distance of LFL by using the dispersion model. The heavy gas dispersion model is choose to simulate LPG dispersion because the molecular weight of LPG is higher than air. 
B\&M (Britter and McQuaid) heavy gas instantaneous dispersion model draws experimental data into nomogram in a dimensionless form.

\section{Risk calculation}

Death probability. Risk is the product of the frequency and the consequence. The likelihood frequency is calculated by the event tree and the potential consequence is calculated by the fatality probability. To acquire the fatality probability, the first step is to use the consequence model to calculate the relationship between thermal radiation, concentration and overpressure with distance, then calculate the probability of death at a certain point.

For BLEVE, the death probability of fireball heat radiation is calculated by Eq. 1 [2].

$$
P_{r}=-36.38+2.56 \ln \left(Q^{4 / 3} \times t\right) \text {. }
$$

Where, $P_{r}(-)$ is the death probability of heat radiation, $Q\left(\mathrm{~W} / \mathrm{m}^{2}\right)$ is the thermal radiation intensity, $t(\mathrm{~s})$ is the exposure time.

For VCE, the death probability is $100 \%$ in the area of over $0.03 \mathrm{MPa}$ overpressure and the death probability is 0 in the area of less than $0.01 \mathrm{MPa}$ overpressure.

For flash fire, the death probability is related to LFL. In the area of flash fire, the death probability is $100 \%$, and the death probability is 0 outside the area of flash fire.

Individual risk. Individual risk is presented by the individual risk contour, calculated by Eq. 2 [3].

$$
I R_{x, y}=\sum_{i=1}^{n} f_{i} P_{f, i}
$$

Where, $I R_{x, y}$ is the individual risk at $(x, y), f_{i}$ is the frequency of scenario $i, P_{f, i}$ is the death probability of scenario $i$ at $(x, y)$.

Risk criteria. To evaluate the actual risk level of facilities, the risk value is compared with the risk criteria. China's risk acceptance criteria refer to the State Administration of Work Safety Decree No. 40 [4] and Notice No. 13 [5]. Individual acceptable risk standards are issued according to three levels of vulnerable objects in accordance with the low density, high density, and special high density.

\section{Case study}

A petrochemical plant storage tank area has five LPG spherical tanks. Parameters are shown in Table 1. The individual risk of LPG tanks is calculated by the software Phast Risk.

Table 1 Parameters of LPG tank

\begin{tabular}{ccccc}
\hline Substance & Volume $\left[\mathrm{m}^{3}\right]$ & Number & Pressure $[$ barg $]$ & Temperature $\left[{ }^{\circ} \mathrm{C}\right]$ \\
\hline LPG & 650 & 5 & 10 & 20 \\
\hline
\end{tabular}

The three severest scenarios of BLEVE, VCE and flash fire are selected. The data of tank failure frequency and leakage aperture are acquired by the standard Based on the Risk Test of the Basic Method. The risk calculation can be completed by the software after input the required data, as well as the risk curve tracking and drawing. The individual risk is set as the upper acceptable limit of operating device according to State Administration of Work Safety Notice No. 13. The calculation results are shown in Fig. 3. 


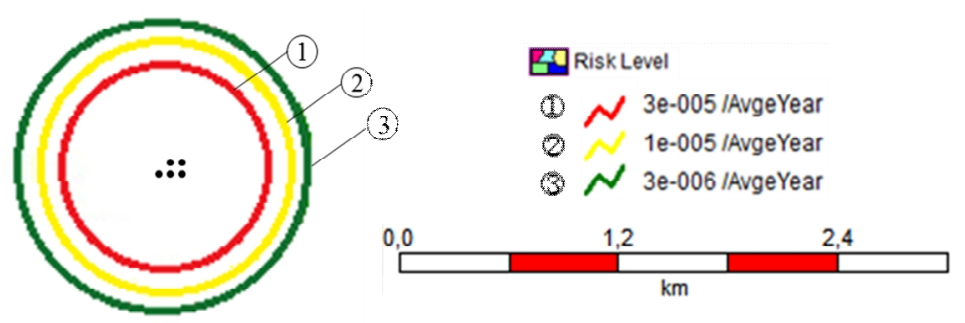

Fig. 3 Individual risk

To follow the risk criteria, the results show that required safety distaneces for vulnerable objects of low density, high density, and special high density should be $600 \mathrm{~m}, 800 \mathrm{~m}$, and $900 \mathrm{~m}$, respectively, shown in Table 2 . When planning the layout of the tank area, reasonable optimization should be carried out in combination with the risk calculation results.

Table 2 Risk calculation results

\begin{tabular}{cccl}
\hline No & $\begin{array}{c}\text { Individual } \\
\text { risk } \\
\text { [/year] }\end{array}$ & $\begin{array}{c}\text { Required } \\
\text { safety } \\
\text { distance }\end{array}$ & Vulnerable objects description \\
\hline 1 & $3 \times 10^{-5}$ & $600 \mathrm{~m}$ & low density places less than 30 people cannot enter the risk contour \\
\hline 2 & $1 \times 10^{-5}$ & $800 \mathrm{~m}$ & high-density places such as residential areas cannot enter the risk contour \\
\hline 3 & $3 \times 10^{-6}$ & $900 \mathrm{~m}$ & special high-density places such as schools cannot enter the risk contour \\
\hline
\end{tabular}

\section{Conclusions}

Based on the QRA of LPG tank leak, the conclusions are as follows:

(1) Analyze the event sequences after LPG tank leak according to historical accidents. The severest consequences are proposed under the condition of different leak holes, which provide suggestions for the risk assessment scenario selection of long-distance vulnerable objects.

(2) The consequence models of LPG tank leak are proposed, which can be used to calculate the thermal radiation damage, overpressure damage of BLEVE, VCE and flash fire. The results provide a theoretical model and application algorithm for determining the explosion hazardous area.

(3) The case study reveals that the key vulnerable objects such as residential areas, schools should keep away from the storage tank areas. The safety distance should be satisfied with requirements of the risk acceptance criteria. The results provide data support from the perspective of risk management for determining the safety distance between chemical plant and the surrounding environment.

\section{Acknowledgements}

This work was financially supported by the National Key Research and Development Program (No. 2016YFC0801500).

\section{References}

[1] H. Pasman and G. Reniers, "Past, present and future of Quantitative Risk Assessment (QRA) and the incentive it obtained from Land-Use Planning (LUP)," J. Loss Prev. Process Ind., vol. 28, pp. 2-9, 2014.

[2] Center for Chemical Process Safety. Guidelines for chemical process quantitative risk analysis [M]. New York: American Institute of Chemical Engineering, 2000.

[3] S. Baesi, B. Abdolhamidzadeh, C. R. C. Hassan, M. D. Hamid, and G. Reniers, "Application of a multi-plant QRA: A case study investigating the risk impact of the construction of a new plant on an existing chemical plant's risk levels," J. Loss Prev. Process Ind., vol. 26, no. 5, pp. 895-903, 2013. 
[4] China State Administration of Work Safety, Interim provisions on supervision and administration of major hazard sources of hazardous chemicals. (Decree No. 40). 2011.

[5] China State Administration of Work Safety, Individual and Societal Acceptable Risk Criteria of Production and Storage Facilities of Hazardous Chemicals (Notice No. 13). 2014. 